December 2021

\title{
Did the European Suspension of the AstraZeneca Vaccine Decrease Vaccine Acceptance During the COVID-19 Pandemic?
}

\author{
Michael Bang Petersen ${ }^{1 *}$, Frederik Jørgensen ${ }^{1}$, Marie Fly Lindholt ${ }^{1}$ \\ ${ }^{1}$ Department of Political Science, Aarhus University, Denmark \\ *Corresponding author: michael@ps.au.dk
}

\begin{abstract}
On March 11, 2021, the AstraZeneca vaccine against COVID-19 was suspended in three Nordic countries and, on subsequent days, in other European countries. Using data on vaccine acceptance in eight Western countries obtained on a daily basis, we show that these decisions - and associated news - decreased public vaccine acceptance in several countries and part of this decrease happened in response to suspensions in other countries. The findings demonstrate the importance of international coordination between health authorities during a pandemic such that local authorities are able to put the decisions of foreign authorities into perspective.
\end{abstract}

Keywords: Vaccine acceptance; COVID-19; side-effects; AstraZeneza suspension; international coordination

Conflict of Interest: None declared.

Funding Statement: The study was funded by The Carlsberg Foundation with grant CF20-0044 to Michael Bang Petersen. The funders had no role in the conduct of this research.

Publication: This manuscript has been published as an open access article in Vaccine: https://doi.org/10.1016/j.vaccine.2021.12.026. Please refer this publication for citation and the final version. 
Within a week of March 2021, a number of countries officially announced that they were temporarily suspending the use of the AstraZeneca-vaccine against COVID-19 after concerns about potential side effects in the form of blood clots. Austria was the first country to raise such concerns. On March 7 , Austria announced that they would suspend a single batch of the vaccine. On March 11, Denmark, Norway and Iceland announced that they suspended the vaccine entirely until they had more evidence on its potential side effects. In the following days, a number of countries took the same decision. These suspensions created headlines worldwide (Vogel \& Kupferschmidt, 2021).

It is well-known that concerns about side effects are significantly associated with vaccine skepticism also in the context of COVID-19 vaccines (Kreps et al., 2020; Motta, 2021; Schwarzinger et al., 2021; Jørgensen et al., 2021; Petersen et al., 2021). Accordingly, there has been raised debates over whether the decision to suspend the AstraZeneca-vaccine in some countries has potential effects on levels of acceptance when it comes to COVID-19 vaccines more generally (Larson \& Broniatowski, 2021). In this manuscript, we raise the specific question of whether the suspension decision of some countries impacted vaccine acceptance in other countries. During an international health crisis such as a pandemic, negative information about side-effects will travel quickly across borders, potentially affecting not just the immediate national audience but also international audiences.

We have continuously collected nationally representative surveys about acceptance of a COVID-19 vaccine across eight Western countries: France, Denmark, Germany, Hungary, Italy, Sweden, United Kingdom and United States (for further description, see Lindholt et al., 2021a). In March, data was collected from March 10 to March 16. On this basis, we are able to assess the impact of the suspension decisions on March 11 and subsequent decisions on public COVID-19 vaccine acceptance by comparing levels of acceptance across different specific dates. This also allows us to examine if the decisions in some countries had spill-over effects on acceptance in other countries. In 
this manuscript, we thus provide a real-world examination of what happens to vaccine acceptance when potential side-effects are massively discussed by authorities and news media.

\section{Materials and Methods}

\section{Data}

The data we use for the present purpose was collected in online survey panels, collected over a week each month from December 2020 to April 2021 with approximately 500 participants per month and country (total $\mathrm{N}=20.519$ ). In each data round, survey respondents were quota sampled to match the population margins on age, gender, and geographic location for each of the eight countries. The data collection was carried out in accordance with Aarhus University's Code of Conduct as well as the Committee Act of the Danish National Committee of Health Research Ethics, which states that "Surveys using questionnaires and interviews that do not involve human biological material (section 14(2) of the Committee Act)" are exempted from approval (https://en.nvk.dk/how-to-notify/what-tonotify).

As our measure of vaccine acceptance, we use agreement with the statement: "If the health authorities advise people like me to get an approved vaccine against the coronavirus, I will follow their advice". Individuals who answer "completely agree" and "somewhat agree" are considered "accepting" and coded as "1". All other responses ("neither agree nor disagree", "somewhat disagree", "completely disagree" and "don't know") are coded as "0".

\section{Research Design}

To identify the causal impact of the suspension decisions and the associated news coverage, we compare levels of vaccine acceptance on March 10 with levels of vaccine acceptance on subsequent days. This strategy is limited by the fact that we are not able to assess the impact of events prior to 
March 10; specifically, Austria's decision to suspend a single batch of the AstraZeneca-vaccine on March 7. However, Figure 1 shows that there is good reason to believe that public attention was not driven by this decision, but mainly generated from the decisions made on March 11. Figure 1 provides an overview of all decisions (vertical dashed lines) by European societies in the focal days around the suspensions together with the development in popular attention about the AstraZeneca vaccine from March 6 to March $18 .{ }^{1}$ While the figure shows a marked increase in relative search volumes in most countries on March 11, there is no increase on March 6. Moreover, we see large country-specific increases in relative search volumes in Italy, Germany, and France on March 15 associated with their decisions to suspend the vaccine.

- Figure 1 here -

This identification strategy has the key strength-compared to alternatives - that it provides a comparison of observations immediately before and after the key suspension event. A previous study that compared rates of vaccine acceptance in Denmark in February (sampled between the $4^{\text {th }}$ and $21^{\text {st }}$ ) and in March (sampled between the $15^{\text {th }}$ and $25^{\text {th }}$ ) found no change in overall vaccine acceptance (Sønderskov et al., 2021). However, the study faces at least two challenges. First, by comparing postsuspension observations to a benchmark that was observed over a month prior to the suspension, estimates will be biased towards zero if vaccine acceptance is in an upwards trend. Figure 2 displays our data setup. Red filled circles reflect the observations that we use in the estimations below to estimate the effect of suspensions on vaccine acceptance, while the blue filled circles show vaccine

\footnotetext{
${ }^{1}$ As a metric of popular attention, we use Google searches for "astrazeneca", accessed via Google Trends. Google Trends provide a relative search volume, which is the query share of a particular term for a given location and time period, normalized by the highest query share of that term over the time-series (see Nuti et al., 2014).
} 
acceptance in previous and later rounds of data. The figure clearly illustrates that vaccine acceptance, in fact, is in an upwards trend from December until March 10 across all countries-except in the United States - in our sample. Potentially this reflects that acceptance is increasing with a successful implementation of the vaccine program. In any case, it implies that comparing post-suspension vaccine acceptance to vaccine acceptance in early February will provide a biased counterfactual (i.e., what we would expect to have happened had there been no suspensions and no reports about potential side effects). Second, if the health communication strategies regarding the suspensions are successful, we might expect a recovery of vaccine acceptance rates after an initial downward drop. In other words, we might expect negative effects on vaccine acceptance to be only temporary. Figure 2 also shows mean vaccine acceptance in April (about a month after the suspensions). Here, after an initial decrease in March, we observe exactly this recovery of acceptance in April. Consequently, it suggests that using post-suspension observations relatively far away from the suspension threshold will similarly create a bias in the estimates toward zero. Taken together, this means that the only way to obtain a credible counterfactual and reliably assess the suspensions' causal impact on vaccine acceptance is to compare vaccine acceptance just around the suspensions.

- Figure 2 here -

\section{Statistical analysis}

We compare the vaccine acceptance proportions before and after the AstraZeneca suspensions (see the red filled circles and vertical dashed lines in Figure 2) using ordinary least squares regression with country-level fixed effects. Our identification strategy thus rests on the assumption that the samples are comparable on different days. Consistent with this, the sampling of the survey was intentionally 
designed to achieve an equal number of responses each day of the week. To adjust for remaining sampling imbalances, all analyses utilize post-stratification weights such that the samples of each day match the population margins on age, gender, education and geographic location.

\section{Results}

Table 1 reports the results of our regression analyses. The first column gives the results from regressing vaccine acceptance on a dummy that indicates the March 11 suspension across all countries. Pooling across all countries, we find that vaccine acceptance fell with an average of 8 percentage points $(b=-.08, p=.002)$ after March 10. In the second and third columns, we split the sample into the countries in the sample that at some point during the analyses period suspended the AstraZeneca vaccine (Denmark, Sweden, Germany, France, and Italy) and the countries that did not suspend the vaccine (Hungary, the United Kingdom, and the United States). Taken together, columns 2 and 3 show that the pooled suspension effect estimate was driven by the countries that decided to suspend the AstraZeneca vaccine within the analyses period. More specifically, pooling across the countries that decided to suspend the AstraZeneca-vaccine the second column shows lower acceptance after March $10(b=-.11, p<.0001)$. At the same time, the third column shows that there was no effect in the countries that did not suspend the vaccine $(b=-.01, p=.845)$. The difference (modeled as an interaction term) between the two groups of countries that did and did not suspend the AstraZeneca vaccine, respectively, is statistically significant $(p=.041)$. In Model IV, displayed in the fourth column, we estimate whether the local suspensions in Germany, France, Italy, and Sweden (see Figure 1 for details) resulted in additional decreases after the national suspensions. Here, we regress vaccine acceptance on a variable with three levels that indicates (1) the period before March 11, (2) the period between March 11 and the local suspensions (reference category), and (3) 
the period after the local suspension. The results show an additional decrease of 7 percentage points $(b=-.07, p=.044)$ after the local national suspension.

- Table 1 here -

\section{Discussion and Conclusion}

These findings suggest that news about the side-effects of the AstraZeneca vaccine and the associated decisions to suspend the vaccine on March 11 had negative, cross-national effects on acceptance of a vaccine against COVID-19 and co-occurred with the ending of a period of increased vaccine acceptance across several countries. The findings thus provide a real-world demonstration of how concerns about side-effects in some countries can depress vaccine acceptance in other countries in the context of the COVID-19 pandemic, creating international ripple effects of national decisions.

Importantly, the identified adverse ripple effects were limited in the sense that they were specific for individuals within Western European countries who are accustomed to relying on shared health information (e.g., from the European Medical Agency). Furthermore, the ripple effects may also be limited in another sense. Unpublished data from another study on vaccine acceptance among Danish respondents also obtained daily data but used a simple "Yes vs. No"-response format for vaccine acceptance. In these data, there is no decrease in vaccine acceptance in the days following the suspension among Danes (Lindholt et al., 2021b). This suggests that the suspensions and associated news may not depress acceptance as such, but rather induce psychological uncertainty about the individual's acceptance; something which is more likely to be captured by the more finegrained measure used here.

It is important to emphasize that findings should not be taken to suggest that authorities should not transparently disclose negative information about vaccines. A recent experimental study 
find that while may such transparency may hinder short-term vaccine acceptance, it can uphold trust in the health authorities (Petersen et al., 2021), which is the biggest predictor of overall acceptance of COVID-19 vaccines (Lindholt et al., 2021). Instead, the contribution from the present analyses is that they provide the first evidence that decisions from one national health authority during a pandemic can reach and impact the populations of other countries. These findings thereby highlight the importance of rapid information sharing and collaboration between national health authorities such that each national authority immediately can qualify the decisions of foreign health authorities and put them into an appropriate national perspective.

\section{Acknowledgements}

We thank Alexander Bor for help with the design and collection of the data. We thank Magnus Storm Rasmussen for help with data management. The data was collected by the survey company, Epinion, on behalf of the researchers. Data and command files are available at Open Science Framework: https://osf.io/knq8e/.

\section{References}

Jørgensen, F. J., Bor, A., \& Petersen, M. (2021, June 28). How the Development, Features and Roll-Out of a SARS-COV-2 Vaccine Shape Public Acceptance: A Conjoint Experiment in a Large Representative Sample of Danes. https://doi.org/10.31234/osf.io/4y8ap

Kreps, S., Prasad, S., Brownstein, J. S., Hswen, Y., Garibaldi, B. T., Zhang, B., \& Kriner, D. L. (2020). Factors associated with US adults' likelihood of accepting COVID-19 vaccination. JAMA network open, 3(10), e2025594-e2025594.

Larson, H. J., \& Broniatowski, D. A. (2021). Volatility of vaccine confidence. Science, 317(6536): 1289. 
Lindholt, M. F., Jørgensen, F. J., Bor, A., \& Petersen, M. B. (2021a). Public Acceptance of COVID-19 Vaccines: Cross-National Evidence on Levels and Individual-Level Predictors Using Observational Data. BMJ Open, in press.

Lindholt, M. F., Jørgensen, F. J., Bor, A., \& Petersen, M. B. (2021b). Danskernes Smitteforebyggende Adfærd og Opfattelser. Report from the HOPE-project, March 15, 2021: https://raw.githubusercontent.com/mariefly/HOPE/master/Danskernes_Smitteforebyggende_ Adfærd_Og_Opfattelser_0316.pdf.

Motta, M. (2021). Can a COVID-19 vaccine live up to Americans' expectations? A conjoint analysis of how vaccine characteristics influence vaccination intentions. Social Science \& Medicine, 272, 113642.

Nuti, S. V., Wayda, B., Ranasinghe, I., Wang, S., Dreyer, R. P., Chen, S. I., \& Murugiah, K. (2014). The use of google trends in health care research: a systematic review. PloS one, 9(10), e109583.

Petersen, M. B., Bor, A., Jørgensen, F. J., \& Lindholt, M. F. (2021). Transparent Communication About Negative Features of COVID-19 Vaccines Decreases Acceptance but Increases Trust. Proceedings of the National Academy of Sciences, in press.

Schwarzinger, M., Watson, V., Arwidson, P., Alla, F., \& Luchini, S. (2021). COVID-19 vaccine hesitancy in a representative working-age population in France: a survey experiment based on vaccine characteristics. The Lancet Public Health, 6(4), e210-e221.

Sønderskov, K. M., Dinesen, P. T., \& Østergaard, S. D. (2021). Sustained COVID-19 vaccine willingness after safety concerns over the Oxford-AstraZeneca vaccine. Dan Med $J, 68$, A03210292.

Vogel, G., \& Kupferschmidt, K. (2021). New problems erode confidence in AstraZeneca's vaccine. Science, 371(6536): 1294-1295. 


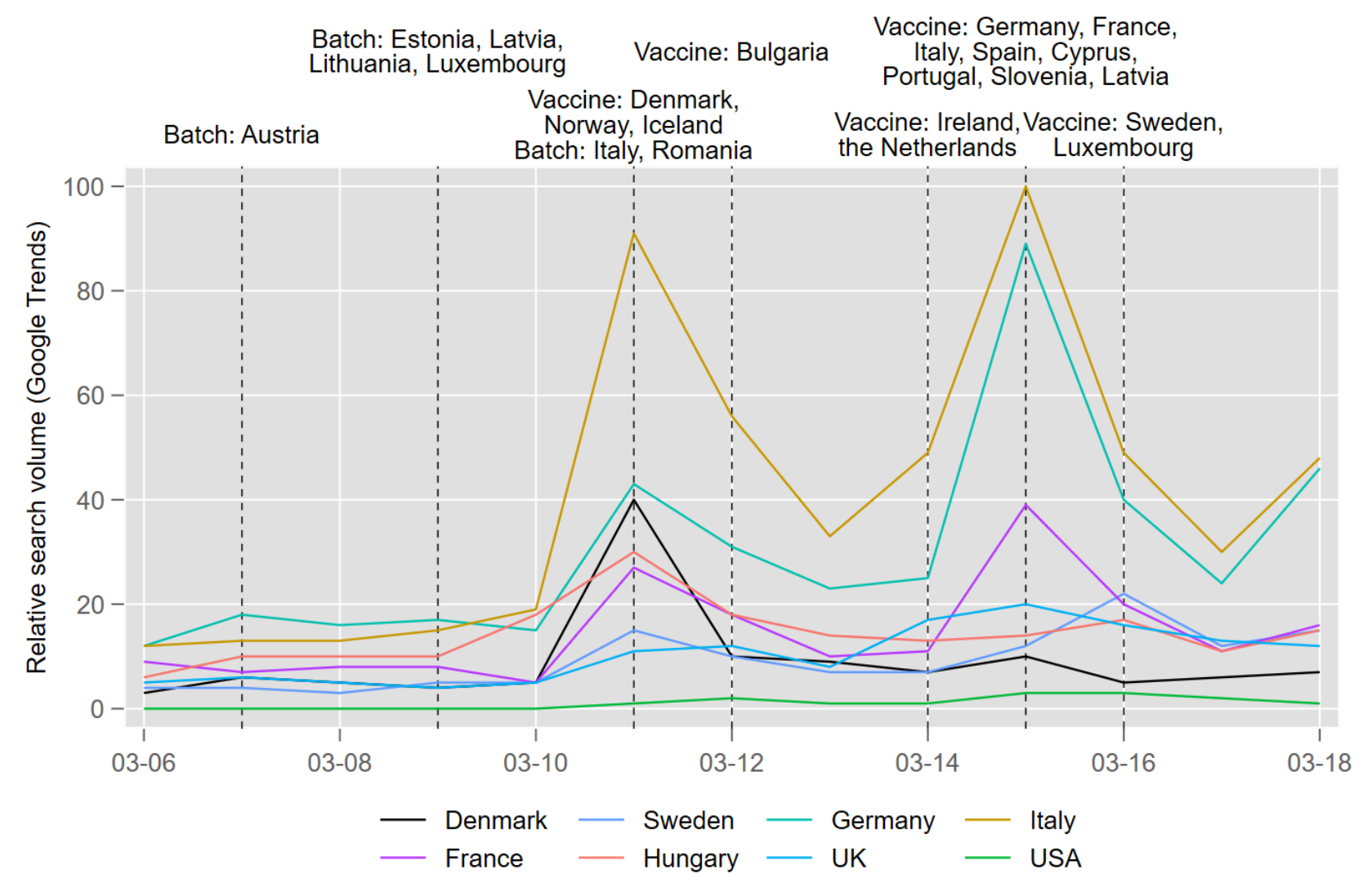

Figure 1. Suspension Events Related to the AstraZeneca Vaccine in Europe and Public Attention to the AstraZeneca Vaccine in the Same Period in Eight Western Democracies. Suspension events are divided into suspensions of single batches ("Batch") and the entire vaccine ("Vaccine"). Dashed vertical lines and associated text indicate the suspension event, the country and the date. Public attention is operationalized as searches on Google for "astrazeneca", measured via Google Trends. 

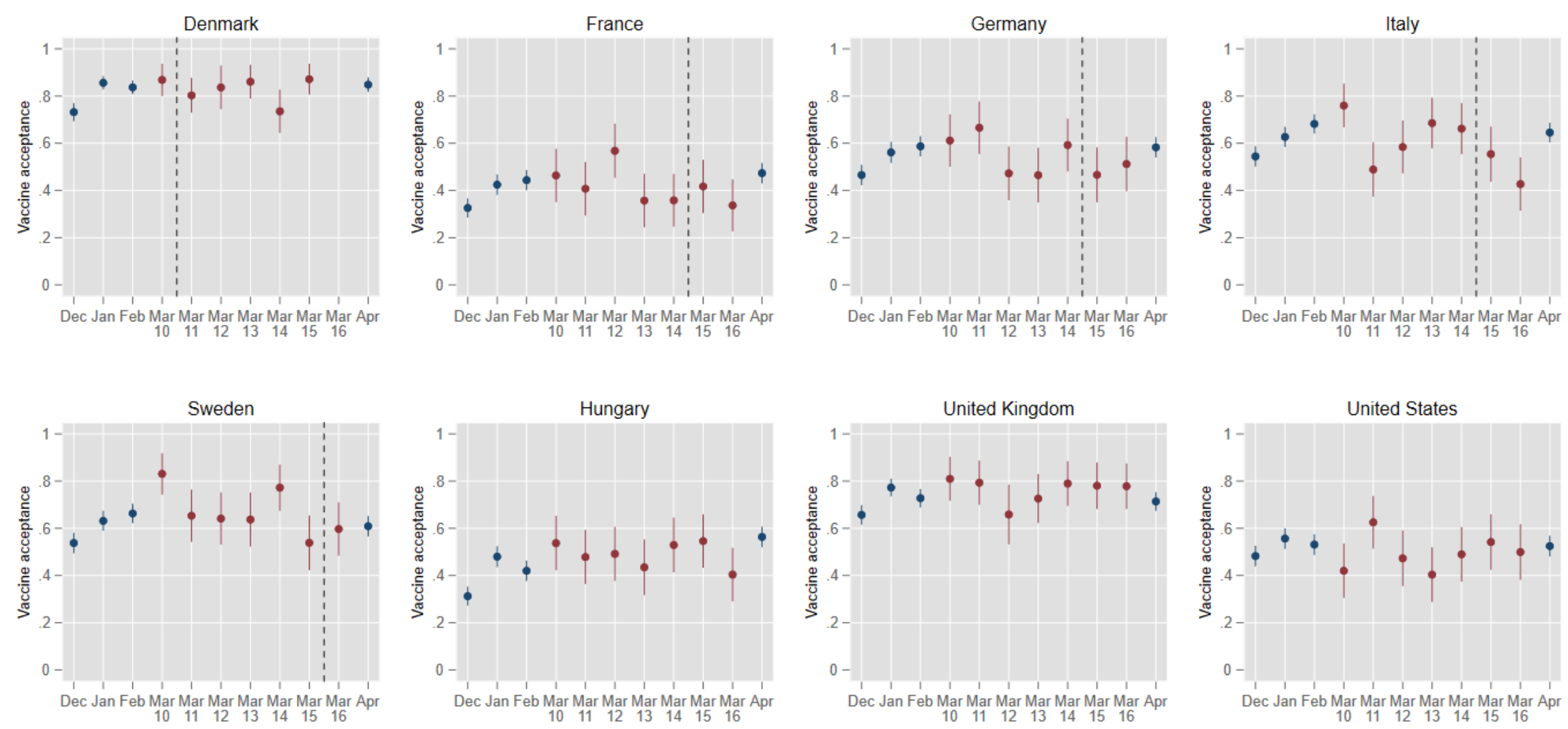

Figure 2. Vaccine Acceptance Across Eight Western Democracies in the Week the AstraZeneca Vaccine Was Suspended. Entries are monthly and daily national proportions (and associated $95 \%$ confidence intervals) who answered "completely agree" or "somewhat agree" when asked whether they were willing to receive an approved COVID-19 vaccine. Red filled circles are the daily vaccine acceptance means from the March data collection that we use in the statistical estimations. Blue filled circles are means from data rounds in months before and after the suspensions, respectively. Vertical dashed lines indicate the day of suspension of the vaccine in each of the countries (Hungary, the UK, and the USA never suspended the AstraZeneca vaccine). There are approximately 500 respondents in each country per month $(\mathrm{N}=$ 20.519). 


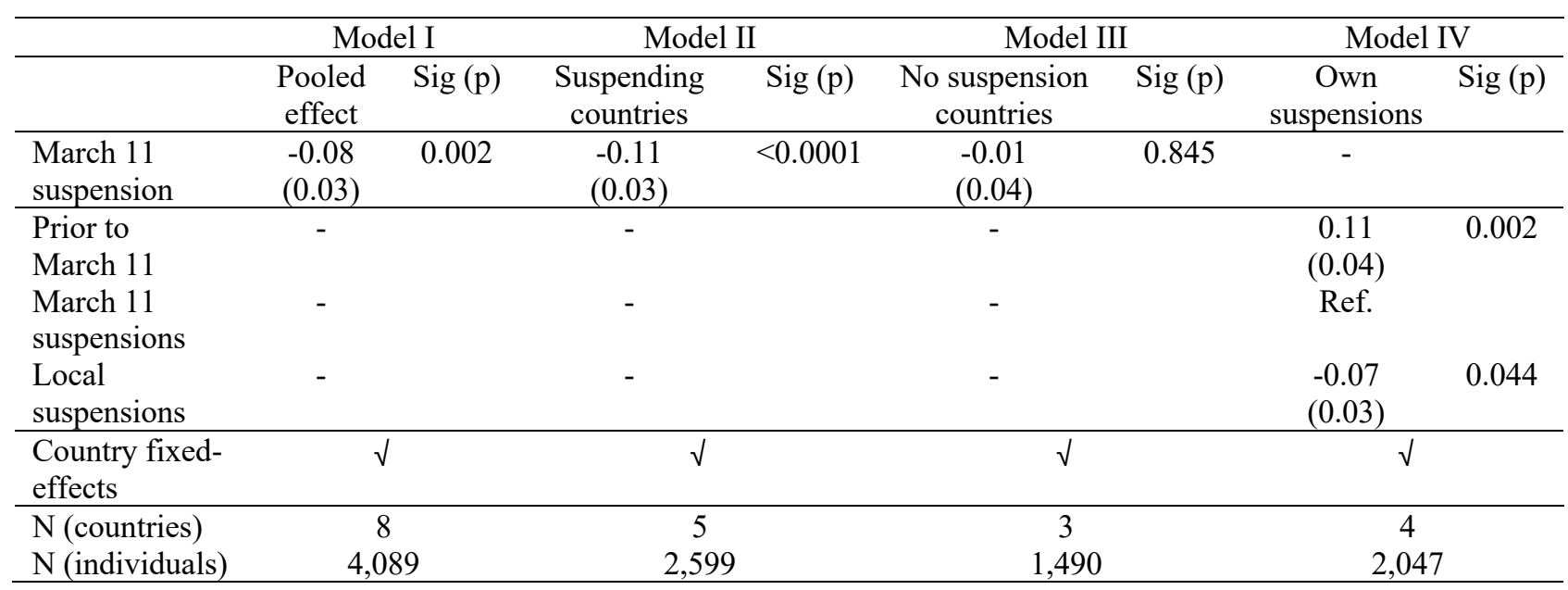

Table 1. Estimated suspension effect on vaccine acceptance. Entries are unstandardized regression coefficients. Robust standard errors in parentheses. Model I includes all countries. Model II includes Denmark, Sweden, France, Germany, and Italy. Model II includes Hungary, the UK, and the USA. Model IV includes Sweden, France, Germany, and Italy. 